\title{
The role of salvage radiotherapy in recurrent thymoma
}

\author{
Andrew Jihoon Yang, Seo Hee Choi, Hwa Kyung Byun, Hyun Ju Kim, \\ Chang Geol Lee, Jaeho Cho \\ Department of Radiation Oncology, Yonsei Cancer Center, Yonsei University College of Medicine, Seoul, Korea
}

Purpose: To explore the role of salvage radiotherapy (RT) for recurrent thymoma as an alternative to surgery.

Materials and Methods: Between 2007 and 2015, 47 patients who received salvage RT for recurrent thymoma at Yonsei Cancer Center were included in this study. Recurrent sites included initial tumor bed $(n=4)$, pleura $(n=19)$, lung parenchyma ( $=10)$, distant $(n=9)$, and multiple regions $(n=5)$. Three-dimensional conformal and intensity-modulated RT were used in 29 and 18 patients, respectively. Median prescribed dose to gross tumor was $52 \mathrm{~Gy}$ (range, 30 to $70 \mathrm{~Gy}$ ), with equivalent doses in 2-Gy fractions $\left(\mathrm{EQD}_{2}\right)$. We investigated overall survival (OS), progression-free survival (PFS), and patterns of failure. Local failure after salvage RT was defined as recurrence at the target volume receiving $>50 \%$ of the prescription dose.

Results: Median follow-up time was 83 months (range, 8 to 299 months). Five-year OS and PFS were 70\% and 22\%, respectively. The overall response rate was 97.9\%; complete response, 34\%; partial response, 44.7\%; and stable disease, 19.1\%. In multivariate analysis, histologic type and salvage RT dose $\left(\geq 52 \mathrm{~Gy}_{1} \mathrm{EQD}_{2}\right.$ ) were significantly associated with $\mathrm{OS}$. The high dose group ( $\geq 52 \mathrm{~Gy}$, $E_{20}$ ) had significantly better outcomes than the low dose group (5-year OS: $80 \%$ vs. 59\%, $p=0.046 ; 5$-year PFS: $30 \%$ vs. $14 \%$, $p=0.002$ ). Treatment failure occurred in 34 patients; out-of-field failure was dominant (intra-thoracic recurrence 35.3\%; extrathoracic recurrence $11.8 \%$ ), while local failure rate was $5.8 \%$.

Conclusion: Salvage RT for recurrent thymoma using high doses and advanced precision techniques produced favorable outcomes, providing evidence that recurrent thymoma is radiosensitive.

Keywords: Thymoma, Radiotherapy, Survival, Recurrence

\section{Introduction}

Thymomas are rare neoplasms that arise in the anterior mediastinum [1]. The rate of thymoma recurrence after thymectomy is $10 \%$ to $29 \%$, and patients diagnosed with thymoma can relapse many years after initial treatment [24]. Because of the relatively small incidence of thymoma, as well as rates of recurrence, delayed recurrence, and variable patterns of recurrence, it is difficult to accurately validate effective management strategies. Some authors have suggested a surgical approach whenever possible in the case of recurrent thymic tumors, reporting good long-term survivals even after reoperation [5]. Others have supported and recommended chemotherapy or radiotherapy $(\mathrm{RT})[3,6]$ (Fig. 1). No significant difference in survival rates between operative and non-operative management strategies have been found, and surgical treatment has been discouraged due to excessive perioperative morbidity $[4,7]$. To date, the lack of

Received 22 January 2019, Revised 09 May 2019, Accepted 27 June 2019.

Correspondence: Jaeho Cho, Department of Radiation Oncology, Yonsei University College of Medicine, 50-1 Yonsei-ro, Seodaemun-gu, Seoul 03722, Korea. Tel: +82-2-2228-8095, Fax: +82-2-312-9033, E-mail: JJHMD@yuhs.ac (http://orcid. org/0000-0001-9966-5157)

(c) This is an Open Access article distributed under the terms of the Creative Commons Attribution Non-Commercial License (http://creativecommons.org/ licenses/by-nc/4.0/) which permits unrestricted non-commercial use, distribution, and reproduction in any medium, provided the original work is properly cited.

www.e-roj.org 

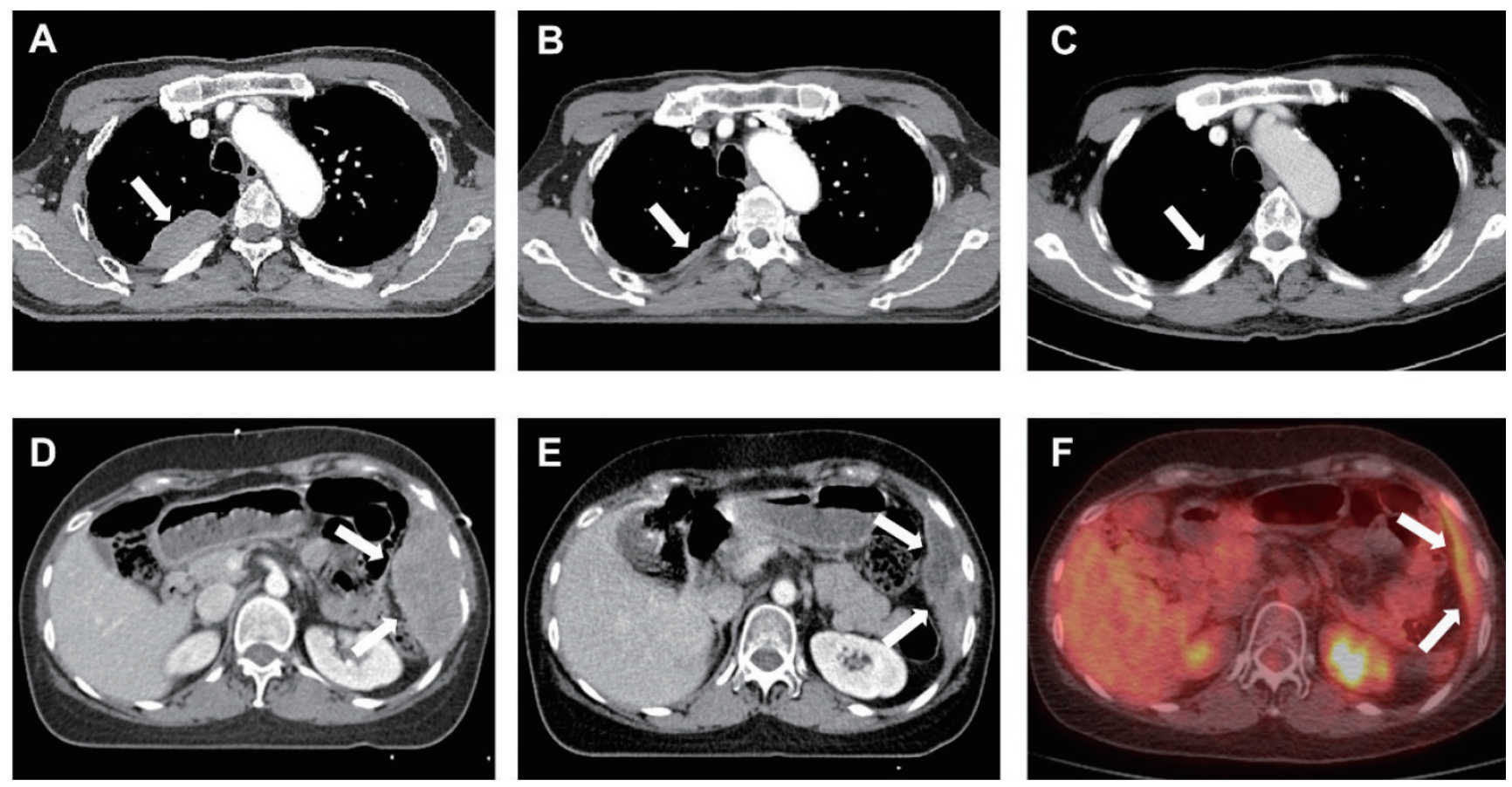

Fig. 1. Excellent response of a recurrent tumor to salvage radiotherapy. (A) Pretreatment simulation computed tomography (CT) scan showing a recurrent tumor (arrow). The patient underwent salvage radiotherapy with a first plan dose of $40 \mathrm{~Gy}$ in 20 fractions. (B) Simulation CT scan during radiotherapy for adaptive planning showing the rapid shrinkage of tumor (arrow). The patient underwent adaptive radiotherapy with a second plan dose of $12 \mathrm{~Gy}$ in 5 fractions. (C) CT scan 3 months after radiotherapy showing the complete remission (arrow). (D) Pretreatment simulation CT scan showing a recurrent tumor (arrow). The patient underwent salvage radiotherapy with a first plan dose of $40 \mathrm{~Gy}$ in 20 fractions. (E) Simulation CT scan during radiotherapy for adaptive planning showing the rapid shrinkage of tumor (arrow). The patient underwent adaptive radiotherapy with a second plan dose of 14 Gy in 7 fractions. (C) Positron emission tomography-computed tomography (PET-CT) scan 3 months after radiotherapy showing the partial remission (arrow).

published data has resulted in a lack of consensus on standard management of recurrent thymoma.

Given the sensitivity of these tumors to radiation, RT has been generally adopted to eradicate potential residual tumor burdens following surgery $[8,9]$, and has become the definitive treatment for unresectable disease [10]. However, the appropriate role of salvage RT in the treatment of recurrent tumors remains unclear. This retrospective study was designed to (1) assess clinical outcomes after RT in treatment of recurrent thymoma, (2) analyze the prognosis factors of recurrent thymoma, and (3) analyze the patterns of failure in recurrent thymoma.

\section{Materials and Methods}

\section{Patients and treatment}

Fifty-eight patients with histologic diagnoses of recurrent thymoma and thymic carcinoma who were treated with salvage RT at Yonsei Cancer Center from August 2007 to
December 2015 were considered for participation in the study. Among these 58 patients, some underwent salvage RT for the first recurrence, and some underwent other treatments for the first few recurrences but continued to have their disease return. For recurrent thymoma, surgery was considered as standard treatment, but salvage RT was performed in patients who had difficult for surgery. To meet the eligibility criteria of salvage RT, we considered patients: (1) with inoperable lesions or multiple lesions, (2) with poor performance status, and (3) who refused surgery. Eleven were excluded: 4 who had no available clinical records, 3 who had incomplete RT of $<20 \mathrm{~Gy}$, and 4 patients who had undergone surgery before RT. As a result, 47 patients were determined eligible for the final analysis. Patients were staged according to the Masaoka staging system. Tumor histology was classified according to the World Health Organization histology classification system. Clinical data at the time of initial diagnosis including age, histology type, initial stage, use of surgery, use of RT, and pathologic findings were collected. In addition, clinical data at 
the time of disease recurrence such as the recurrence interval, recurrence site, number of recurrent lesion, RT modality, RT dose, and use of chemotherapy, were collected. After salvage RT was performed, treatment outcomes were evaluated. To account for various fractionation schedules, all calculations regarding radiation dose effects were performed using the equivalent dose in $2-G y$ fractions $\left(E_{0 D}\right)$ with an $\alpha / \beta$ value of 10. The informed consent was waived.

\section{Follow-up assessment and patterns of failure}

Patients were assessed using chest computed tomography 1 month after completing RT, and afterwards, generally every 3 to 6 months. Patterns of failure were classified according to relative location between the recurrence site and radiation isodose curve. In-field recurrence was defined as recurrence that occurred where the radiation dose was $100 \%$ or more. Marginal recurrence was defined as recurrence that occurred where the radiation dose was $<100 \%$ and $>50 \%$. Out-of-field recurrence was defined as intrathoracic recurrence outside the 50\% isodose curve. Distant recurrence was defined as recurrence outside the thoracic region [11]. In addition, tumor responses 3 months after RT were evaluated as complete responses, partial responses, stable disease, and progressive disease using the modified Response Evaluation Criteria in Solid Tumors.

\section{Statistical analysis}

Progression-free survival (PFS) was calculated from the date of first progression to the date of next progression or death. Overall survival (OS) was calculated from the date of progression to the date of the patient's death. In the absence of either a second progression or death, patients were assessed according to the date of the last follow-up visit. Both OS and PFS rates were calculated using the Kaplan-Meier method, and significance of differences in survival rates was assessed using a log-rank test. Multivariate Cox regression analysis was used to identify independent prognostic factors. A chi-square test was used to compare categorical variables, and a t-test was used to compare continuous variables. A $p$-value $<0.05$ was considered statistically significant. SPSS version 23 (IBM, Armonk, NY, USA) software was used for all statistical analyses.

\section{Results}

\section{Patients and treatments}

Patient and treatment characteristics at the time of initial diagnosis are described in Table 1. Median age was 56 years
Table 1. Patient and tumor characteristics at initial diagnosis

\begin{tabular}{|c|c|}
\hline Characteristic & Value \\
\hline Age (yr) & $56(27-87)$ \\
\hline \multicolumn{2}{|l|}{ Sex } \\
\hline Male & $29(61.7)$ \\
\hline Female & 18 (38.3) \\
\hline \multicolumn{2}{|l|}{ Histology } \\
\hline Type B2 & $12(25.5)$ \\
\hline Type B3 & $10(21.3)$ \\
\hline Type C & $23(48.9)$ \\
\hline Unknown & $2(4.2)$ \\
\hline \multicolumn{2}{|l|}{ Initial tumor stage } \\
\hline I & $3(6.4)$ \\
\hline$\| A$ & $6(12.8)$ \\
\hline \multicolumn{2}{|l|}{$\| \mathrm{B}$} \\
\hline III & $11(23.4)$ \\
\hline IVA & $11(23.4)$ \\
\hline IVB & $9(19.1)$ \\
\hline Unknown & $6(12.8)$ \\
\hline \multicolumn{2}{|l|}{ Tumor size $(\mathrm{cm})$} \\
\hline$<5$ & $7(14.9)$ \\
\hline $5-9.9$ & $23(48.9)$ \\
\hline$\geq 10$ & $8(17.1)$ \\
\hline Unknown & 9 (19.1) \\
\hline \multicolumn{2}{|l|}{ Initial treatment } \\
\hline Surgery + adjuvant RT & $29(61.7)$ \\
\hline Surgery alone & $9(19.1)$ \\
\hline Chemotherapy & $6(12.8)$ \\
\hline Chemoradiotherapy & $2(4.2)$ \\
\hline RT alone & $1(2.1)$ \\
\hline \multicolumn{2}{|l|}{ Resection margin } \\
\hline Ro & $15(39.5)$ \\
\hline R1 & $6(15.8)$ \\
\hline $\mathrm{R} 2$ & $8(21.1)$ \\
\hline Unknown & $9(24.3)$ \\
\hline \multicolumn{2}{|l|}{ RT technique } \\
\hline 3D CRT & 30 (93.8) \\
\hline IMRT & $2(6.2)$ \\
\hline \multicolumn{2}{|l|}{ RT dose $\left(E Q D_{2}\right)$} \\
\hline$<54$ Gy & $12(37.5)$ \\
\hline$\geq 54$ Gy & $18(56.3)$ \\
\hline Unknown & $2(6.2)$ \\
\hline
\end{tabular}

Values are presented as median (range) or number (\%). $R T$, radiotherapy; 3D CRT, three-dimensional conformal radiotherapy; IMRT, intensity-modulated radiotherapy; EOD 2 , equivalent dose in 2-Gy fractions. 
(range, 27 to 87 years). The most frequent histologic type at initial diagnosis was type $C_{\text {, accounting for }} 23$ cases (49\%), 12 patients (26\%) had type B2, and 10 patients (21\%) had type B3. The most common initial treatment was surgery with adjuvant RT, accounting for 29 cases (62\%), followed by surgery only (19\%), and chemotherapy (13\%). During initial surgery, Ro resection was achieved in 15 patients (40\%), while R1 and R2 resection were achieved in 6 patients (16\%) and 8 patients (21\%), respectively. Among the patients who underwent RT for their initial treatment, most received threedimensional conformal RT (94\%).

Patient and treatment characteristics at the time of disease recurrence are described in Table 2. The median time interval from initial diagnosis to recurrence was 30 months (range, 1 to 223 months). The most common recurrent site was pleura, accounting for 19 cases (40\%), followed by lung parenchyme (21\%), and extrathoracic sites (19\%). Twenty-nine patients (62\%) underwent salvage RT using three-dimensional conformal RT, while 18 patients (38\%) received salvage RT using intensity-modulated RT. The salvage RT field surrounded the gross tumor, and the planning target volume was defined using a $3 \mathrm{~mm}$ wide margin around the gross tumor volume. The salvage RT dose in $\mathrm{EOD}_{2}$ prescribed to treat tumors varied from a minimum of $30 \mathrm{~Gy}$ to a maximum of $70 \mathrm{~Gy}$ with a median of 52 Gy. Sixteen patients (34\%) underwent additional chemotherapy. The retrospective protocol was approved by our institutional review board. The informed consent was waived.

\section{Survival analysis}

Median follow-up time was 83 months (range, 8 to 299 months). Seventeen of 47 patients (36\%) had died by the time of analysis. All 17 deaths were caused by the progression of thymic malignancy. The most common cause of death was respiratory failure as a consequence of progressive intrathoracic disease $(n=15)$. The other causes were septic shock $(n=1)$ and brain hemorrhage $(n=1)$. Median OS of all patients was 45 months, and median PFS was 23 months. The 5-year OS and PFS for all patients were 70\% and 22\%, respectively (Fig. 2).

\section{Response and pattern of failure}

Table 3 shows response at 3 months after salvage RT in all 47 patients. The overall response rate was $97.9 \%$. Complete response was found in 18 patients (38.2\%), partial response was found in 19 patients (40.5\%), and stable disease was found in 9 patients (19.1\%).

Table 4 shows the patterns of failure after salvage RT in
Table 2. Clinical and treatment characteristics at the time of disease recurrence

\begin{tabular}{lc}
\hline \multicolumn{1}{c}{ Characteristic } & Value \\
\hline $\begin{array}{l}\text { Recurrence interval }(\mathrm{mo}) \\
<30\end{array}$ & $30(1-223)$ \\
$\geq 30$ & $25(53.2)$ \\
Recurrent tumor histology & $22(46.8)$ \\
Type B2 & \\
Type B3 & $7(14.9)$ \\
Type C & $4(8.5)$ \\
Unknown & $5(10.6)$ \\
Recures
\end{tabular}

Recurrence site

Initial tumor bed

Pleura

$19(40.4)$

Lung parenchyme

$10(21.3)$

Extrathoracic metastasis

$9(19.1)$

Multiple

$5(10.6)$

Recurrence multiplicity

Single $\quad 7(14.9)$

Multiple (intrathoracic) 31 (66.0)

Multiple (systemic) $\quad 9$ (19.1)

RT modality

3D CRT $29(61.7)$

IMRT 18 (38.3)

RT dose $\left(\mathrm{EQD}_{2}\right)$

$<52 \mathrm{~Gy} \quad 22(46.8)$

$\geq 52 \mathrm{~Gy} \quad 25(53.2)$

Chemotherapy

No 16 (34.0)

Yes $31(66.0)$

Values are presented as median (range) or number (\%).

$\mathrm{RT}$, radiotherapy; $3 \mathrm{D} C R T$, three-dimensional conformal radiotherapy; IMRT, intensity-modulated radiotherapy; EQD ${ }_{21}$ equivalent dose in 2-Gy fractions.

34 patients who had a second recurrence. Relapses were found at the following sites: in-field (2 patients), marginal (3), intrathoracic out-of-field (23), and distant (6). The most frequent relapse pattern after salvage RT was intrathoracic out-of-field, accounting for $55.8 \%$ of all failures. In contrast, the in-field failure rate accounted for only $5.8 \%$ of all failures, including the location of mediastinum ( 1 patient) and sacrum (1). Distant failure was observed in liver (2 patients), kidney (1), brain (1), rectum (1), and multiple sites (1).

\section{Prognostic factors}

https://doi.org/10.3857/roj.2019.00066 
A

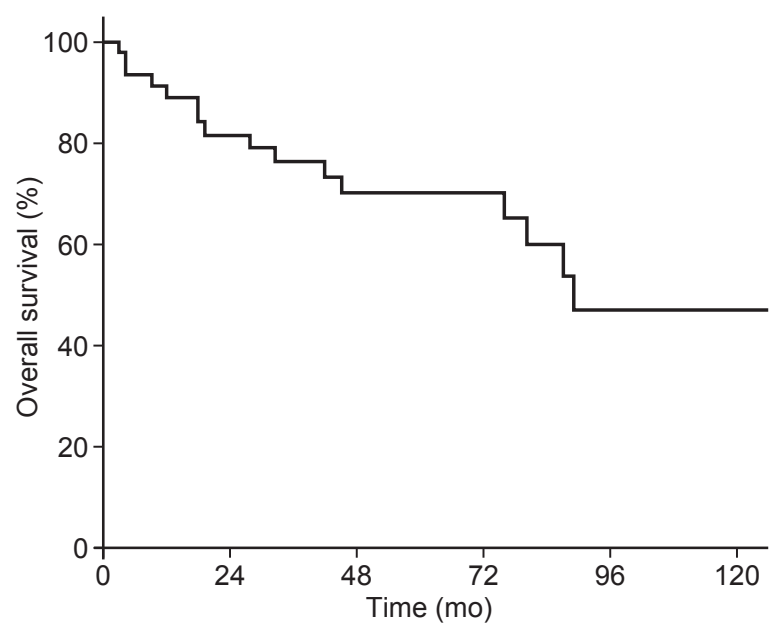

B

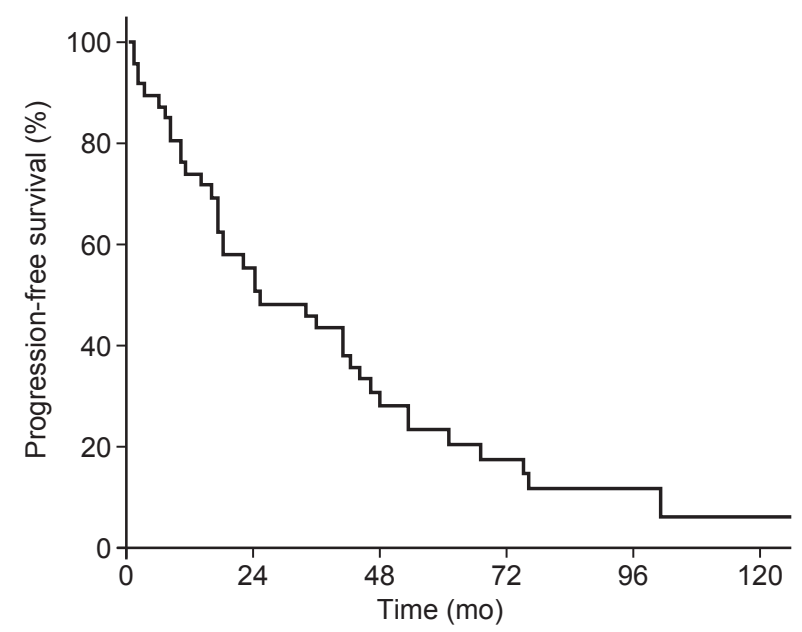

Fig. 2. Kaplan-Meier estimates of (A) overall survival and (B) progression-free survival.

In univariate analyses, histology, initial treatment, and RT dose were significantly associated with OS. Histology, initial stage, initial treatment, recurrent site, and RT dose were significantly associated with PFS (all $p<0.05$ ). After multivariate analysis, high dose remained independently associated with improved OS (hazard ratio $[H R]=0.32 ; 95 \%$ confidence interval [CI], 0.10-0.98; $p=0.046$ ), while type $C$ thymoma remained independently associated with poor OS (HR $=6.70 ; 95 \% \mathrm{Cl}, 2.05-21.8 ; p=0.002)$. High dose also remained independently associated with improved PFS (HR $=0.35$; 95\% Cl, 0.16-0.75; $p=0.007)$, and type $C$ thymoma remained independently associated with poor PFS $(\mathrm{HR}=3.82 ; 95 \% \mathrm{Cl}$, 1.76-8.26; $p=0.001$ ) (Table 5).

When patients were divided into high $\left(\mathrm{EQD}_{2} \geq 52 \mathrm{~Gy}, \mathrm{n}=\right.$ $25)$ and low $\left(\mathrm{EOD}_{2}<52 \mathrm{~Gy}, \mathrm{n}=22\right)$ dose groups, the median survival rates were 49 months (high) and 39 months (low). The 5-year OS rates were 80\% (high dose group) and 59\% (low dose group) ( $p=0.043$ ). The median PFS of the high dose group was 26 months, and 18 months for the low dose group. The 5-year PFS rates were 30\% (high dose group) and 14\% (low dose group) ( $p=0.016$; Fig. 3 ).

\section{Discussion and Conclusion}

Thymoma is known to recur several years after surgery in $10 \%-30 \%$ of patients [2-4]. Some authors recommend reoperation for the treatment of recurrent thymoma, and have demonstrated better survival rates in the complete reresection group $[5,12]$. However, complete resection may not be possible in some cases, due to intimacy with vital structures
Table 3. Treatment response at 3 months after salvage radiotherapy

\begin{tabular}{lc}
\hline \multicolumn{1}{c}{ Response } & No. (\%) \\
\hline Complete response & $18(38.2)$ \\
Partial response & $19(40.5)$ \\
Stable disease & $9(19.1)$ \\
Progressive disease & $1(2.1)$ \\
\hline
\end{tabular}

Table 4. Patterns of failure after salvage radiotherapy

\begin{tabular}{lc}
\hline \multicolumn{1}{c}{ Patterns of failure } & No. $(\%)$ \\
\hline In-field & $2(5.8)$ \\
Marginal & $7(20.6)$ \\
Out-of-field (intrathoracic) & $19(55.8)$ \\
Distant & $6(17.6)$ \\
\hline
\end{tabular}

or surgical morbidity. According to the literature, the operative mortality of recurrent thymoma ranges from $0 \%$ to $13.3 \%$, and the operative morbidity ranges from $0 \%$ to $32.1 \%$ [13]. Thus, some authors recommend the use of RT or chemotherapy over surgery $[3,6]$. To date, standard treatment for recurrent thymoma has not been established, which is not surprising, given the low incidence of thymoma and its relatively indolent biological behavior. Currently, the highest level of knowledge is based on a meta-analysis of 11 retrospective studies, which compared patients treated surgically to those treated nonsurgically [14]. This review suggested that surgery is superior to non-surgical treatment. However, patients in the nonsurgical group had both heterogeneous characteristics and 


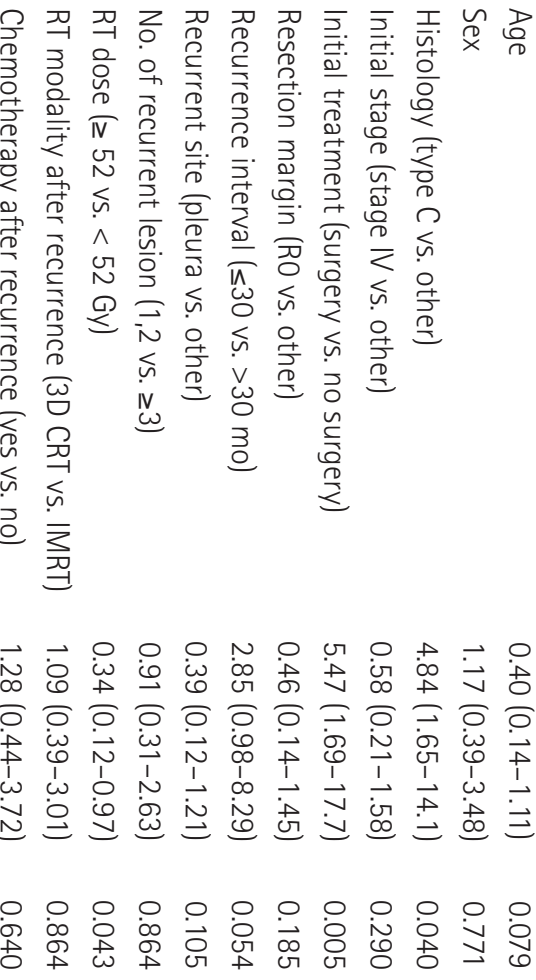

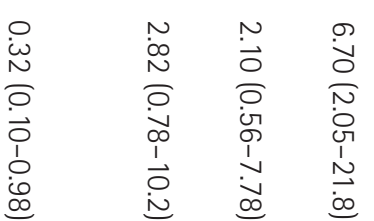

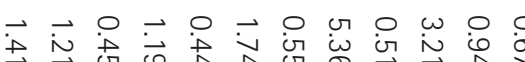

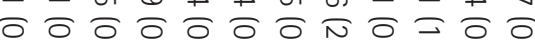

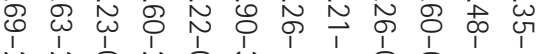

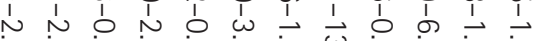

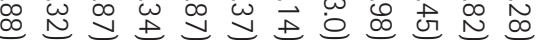

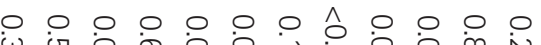
ఉ ज ज

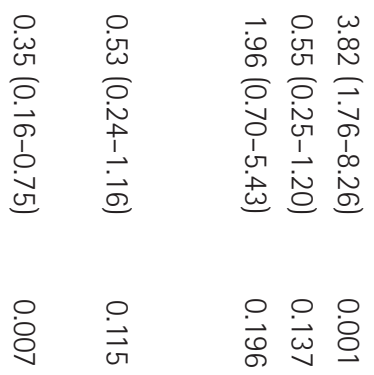

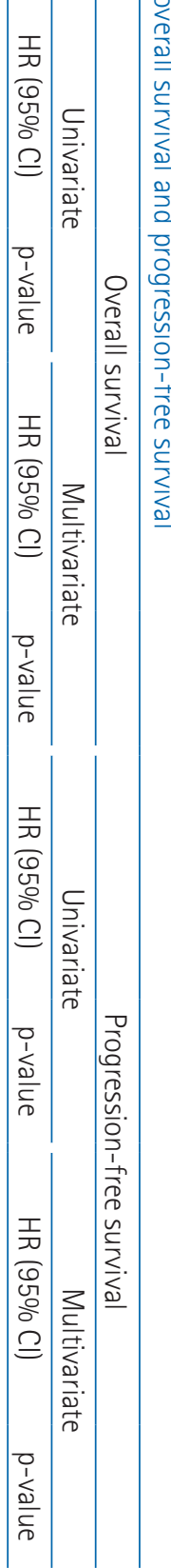

heterogenous treatment modalities, including chemotherapy and/or RT. Also, there may have been a selection bias towards patients who were difficult to conduct surgery due to problems such as performance status and multiplicity [15].

Study participants included patients who underwent postoperative RT $(n=29)$, surgery alone $(n=9)$, chemotherapy alone $(n=6)$, chemoradiotherapy $(n=2)$, and RT alone $(n=1)$ as their initial treatments. However, we do not believe these differences in initial treatment biased our results because the areas that were subjected to salvage RT were RT-naïve. Ultimately, the purpose of this study was to determine the efficacy of salvage RT for recurrent thymoma. Because none of the various initial treatments exhibited a significant correlation with outcomes, all patients-including postoperative RT, surgery alone, chemotherapy alone, chemoradiotherapy and RT alone-were included and analyzed.

In this study, we demonstrated favorable oncologic outcomes with salvage RT. Patients with recurrent thymoma had an excellent response rate (98\%) with salvage RT. Moreover, the 5 -year OS of the high RT dose group was $80 \%$, which was comparable to the reported outcome of patients who received a complete resection after tumor recurrence [16]. Out in several earlier reports imply that thymoma is a radiosensitive tumor [17-20].

In the present study, we focused on patterns of failure in relation to the RT field. Because there are currently few reports on patterns of failure in recurrent thymoma, due to the low prevalence of the disease, we classified the recurrence pattern according to the isodose curve of the salvage RT plan. As a result, the in-field failure rate was only $5.8 \%$ and the marginal failure rate was $8.8 \%$. Given the excellent local control rates, our results suggest that salvage RT may be an effective treatment for recurrent thymoma.

The two independent prognostic factors in our investigation were histology and RT dose. Tumor histology is a well-known prognostic factor of thymoma [21]. RT dose is independently associated with OS and PFS. Thus, one could consider the high-dose RT group the curative group and the low-dose RT group the palliative group. However, in patients with recurrent thymoma, high doses are clearly associated with effective treatment given that dose emerged as a significant factor during the multivariate analysis and appeared related to the number of recurrent lesions that developed. This RT doseresponse relationship supports the idea of the radiosensitive nature of thymoma, as shown previously [18-21], along with the excellent response rate of $98 \%$ and rare in-field recurrence in this study. In the modern RT era, RT dose escalation is 
A

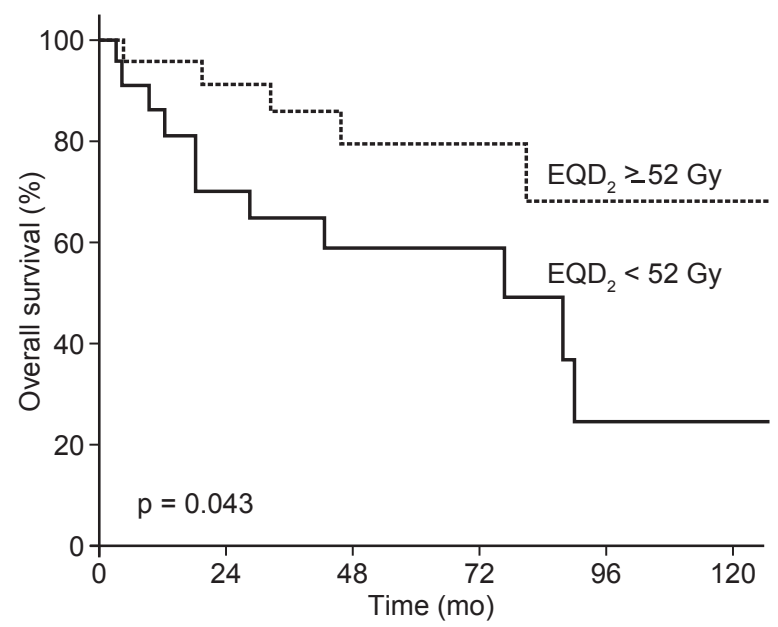

B

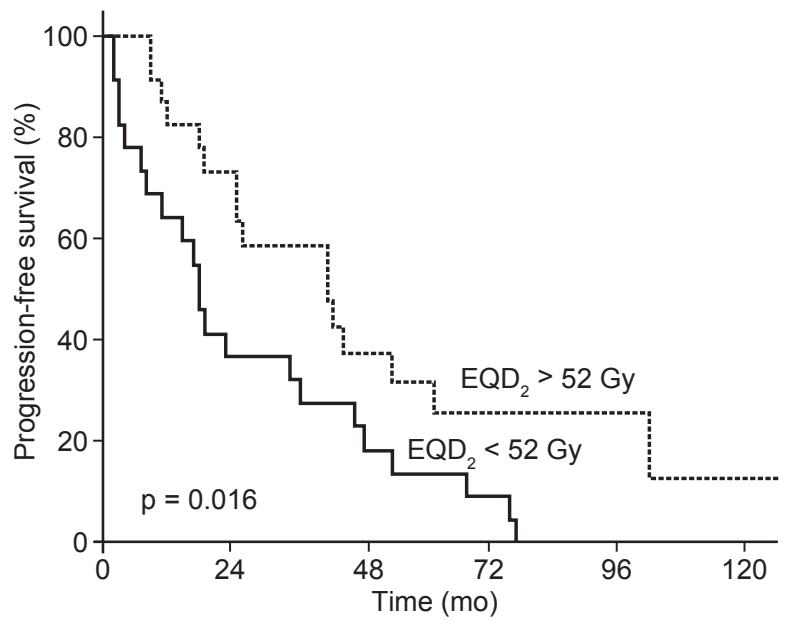

Fig. 3. Kaplan-Meier estimates of (A) overall survival and (B) progression-free survival according to the high ( $>52$ Gy) and low ( $\leq 52$ Gy) dose groups. EQD 2 , equivalent dose in 2-Gy fractions.

easier to achieve through the use of advanced RT technology. In our institution for example, four-dimensional computed tomography-based planning is performed that depicts the tumor and normal organ movement in every respiratory phase. Daily image guidance is performed before every treatment session to ensure accurate localization. These technologies allow increases in the dose delivered to the tumor, while satisfying normal organ dose constraints, making the delivery of higher RT doses feasible.

This study has several limitations. First, it uses a retrospective design and has a relatively small sample size. Second, we did not compare patients who underwent salvage RT to patients who only underwent salvage surgery. Third, RT dose regimens were not consistent, as they were selected according to the recurrent site or with the physician's discretion. In addition, the patients underwent a variety of initial treatments, and therefore, the patient pool was somewhat heterogeneous. Therefore, our findings should be interpreted with some caution. Finally, for patients with multiple lesions, the decision to treat all lesions, or only the symptomatic lesions, was at the discretion of the physician. Some patients exhibited a complete response after initial treatment, whereas other experienced a partial response. There were also patients who underwent radiation therapy at the time of the first recurrence and some patients who underwent radiation therapy only after the second or subsequent recurrence. All of these factors promoted heterogeneity in the study sample.

This paper also has several strengths. First, there is a very low prevalence of thymoma, and its recurrence is a very rare event. This means that many clinicians have limited experience with RT as a treatment for this cancer. Forty-seven patients is a relatively small sample, but considering the rarity of recurrent thymoma, it would be very difficult to design and conduct prospective clinical trials that could adequately evaluate optimal treatment options. Our retrospective cohort treated with salvage RT for recurrent thymoma provides useful information for clinicians. Second, this study was designed, carried out, and reported by radiation oncologists, unlike most previous investigations which were conducted and published by surgeons. This has led to a limited interpretation of RT, especially in terms of radiation dose and failure patterns in relation to the RT field. In contrast, our study analyzed the effect of detailed RT related-parameters such as modality, dose, and field.

In conclusion, we analyzed outcomes from the use of salvage RT in treatment of recurrent thymoma and demonstrated excellent response rates. We also demonstrated that an RT dose of $\mathrm{EQD}_{2} \geq 52$ Gy was associated with better PFS and OS. Our findings suggest that high-dose RT is an effective salvage treatment option for recurrent thymoma. Application of high-dose RT should be a recommended option in the modern RT era, in order to improve oncologic outcomes in cases of recurrent thymoma.

\section{Conflict of Interest}

No potential conflict of interest relevant to this article was 
reported.

\section{References}

1. Macchiarini $P$, Ostertag $H$. Uncommon primary mediastinal tumours. Lancet Oncol 2004;5:107-18.

2. Margaritora S, Cesario A, Cusumano G, et al. Single-centre 40year results of redo operation for recurrent thymomas. Eur J Cardiothorac Surg 2011;40:894-900.

3. Bott MJ, Wang $H_{1}$ Travis W, et al. Management and outcomes of relapse after treatment for thymoma and thymic carcinoma. Ann Thorac Surg 2011;92:1984-2.

4. Haniuda $M$, Kondo R, Numanami H, Makiuchi A, Machida $E$, Amano J. Recurrence of thymoma: clinicopathological features, re-operation, and outcome. J Surg Oncol 2001;78:183-8.

5. Sandri $A$, Cusumano $G$, Lococo $F$, et al. Long-term results after treatment for recurrent thymoma: a multicenter analysis. J Thorac Oncol 2014;9:1796-804.

6. Loehrer PJ Sr, Kim K, Aisner SC, et al. Cisplatin plus doxorubicin plus cyclophosphamide in metastatic or recurrent thymoma: final results of an intergroup trial. The Eastern Cooperative Oncology Group, Southwest Oncology Group, and Southeastern Cancer Study Group. J Clin Oncol 1994;12:11648.

7. Urgesi A, Monetti U, Rossi G, Ricardi U, Maggi G, Sannazzari $\mathrm{GL}$. Aggressive treatment of intrathoracic recurrences of thymoma. Radiother Oncol 1992;24:221-5.

8. Fuller CD, Ramahi EH, Aherne N, Eng TY, Thomas CR Jr. Radiotherapy for thymic neoplasms. J Thorac Oncol 2010;5(10 Suppl 4):S327-35.

9. Rimner A, Yao $X$, Huang J, et al. Postoperative radiation therapy is associated with longer overall survival in completely resected stage II and III thymoma: an analysis of the International Thymic Malignancies Interest Group Retrospective Database. J Thorac Oncol 2016;11:1785-92.

10. Vogel J, Berman AT, Lin L, et al. Prospective study of proton beam radiation therapy for adjuvant and definitive treatment of thymoma and thymic carcinoma: Early response and toxicity assessment. Radiother Oncol 2016;118:504-9.

11. Rimner A, Gomez DR, Wu AJ, et al. Failure patterns relative to radiation treatment fields for stage II-IV thymoma. J Thorac Oncol 2014;9:403-9.

12. Regnard JF, Zinzindohoue F, Magdeleinat $P$, Guibert $L$, Spaggiari $L$, Levasseur P. Results of re-resection for recurrent thymomas. Ann Thorac Surg 1997;64:1593-8.

13. Dai J, Song $N$, Yang $Y$, Jiang G. Is it valuable and safe to perform reoperation for recurrent thymoma? Interact Cardiovasc Thorac Surg 2015;21:526-31.

14. Hamaji M, Ali SO, Burt BM. A meta-analysis of surgical versus nonsurgical management of recurrent thymoma. Ann Thorac Surg 2014;98:748-55.

15. Yano M, Sasaki $H$, Moriyama $S$, et al. Number of recurrent lesions is a prognostic factor in recurrent thymoma. Interact Cardiovasc Thorac Surg 2011;13:21-4.

16. Bae MK, Byun CS, Lee CY, et al. Clinical outcomes and prognosis of recurrent thymoma management. J Thorac Oncol 2012;7:1304-14.

17. Ogawa K, Toita $T$, Uno $T$, et al. Treatment and prognosis of thymic carcinoma: a retrospective analysis of 40 cases. Cancer 2002;94:3115-9.

18. Weksler B, Shende M, Nason KS, Gallagher A, Ferson PF, Pennathur $A$. The role of adjuvant radiation therapy for resected stage III thymoma: a population-based study. Ann Thorac Surg 2012;93:1822-9.

19. Patel S, Macdonald OK, Nagda S, Bittner N, Suntharalingam M. Evaluation of the role of radiation therapy in the management of malignant thymoma. Int J Radiat Oncol Biol Phys 2012;82:1797-801.

20. Chang JH, Kim HJ, Wu HG, Kim JH, Kim YT. Postoperative radiotherapy for completely resected stage II or III thymoma. J Thorac Oncol 2011;6:1282-6.

21. Strobel $P$, Bauer $A$, Puppe $B$, et al. Tumor recurrence and survival in patients treated for thymomas and thymic squamous cell carcinomas: a retrospective analysis. J Clin Oncol 2004;22:1501-9. 